Modelling

Elsevier Editorial System(tm) for Economic

Manuscript Draft

Manuscript Number: ECMODE-D-16-00037R2

Title: A large CVaR-based portfolio selection model with weight constraints

Article Type: Full Length Article

Keywords: Finance; CVaR-based portfolio; risk assessment; weight constraints; quantile regression

Corresponding Author: Dr. Qifa Xu, Ph.D.

Corresponding Author's Institution: Hefei University of Technology

First Author: Qifa Xu, Ph.D.

Order of Authors: Qifa Xu, Ph.D.; Yingying Zhou; Cuixia Jiang; Keming Yu; Xufeng $\mathrm{Niu}$

Abstract: Although the traditional CVaR-based portfolio methods are successfully used in practice, the size of a portfolio with thousands of assets makes optimizing them difficult, if not impossible to solve. In this article we introduce a large CVaR-based portfolio selection method by imposing weight constraints on the standard CVaR-based portfolio selection model, which effectively avoids extreme positions often emerging in traditional methods. We propose to solve the large CVaR-based portfolio model with weight constraints using penalized quantile regression techniques, which overcomes the difficulties of large scale optimization in traditional methods. We illustrate the method via empirical analysis of optimal portfolios on Shanghai and Shenzhen 300 (HS300) index and Shanghai Stock Exchange Composite (SSEC) index of China. The empirical results show that our method is efficient to solve a large portfolio selection and performs well in dispersing tail risk of a portfolio by only using a small amount of financial assets. 


\title{
A large CVaR-based portfolio selection model with weight constraints
}

\author{
Qifa Xu ${ }^{\mathrm{a}, \mathrm{b}, *}$, Yingying Zhou ${ }^{\mathrm{a}}$, Cuixia Jiang ${ }^{\mathrm{a}}$, Keming $\mathrm{Yu}^{\mathrm{c}}$, Xufeng Niu ${ }^{\mathrm{d}}$ \\ ${ }^{a}$ School of Management, Hefei University of Technology, Hefei 230009, Anhui, PR China \\ ${ }^{b}$ Key Laboratory of Process Optimization and Intelligent Decision-making, Ministry of \\ Education, Hefei 230009, Anhui, PR China \\ ${ }^{c}$ Department of Mathematics, Brunel University, Uxbridge, UB8 3PH, UK \\ ${ }^{d}$ Department of statistics, Florida State University, Tallahassee 32304, USA
}

\begin{abstract}
Although the traditional CVaR-based portfolio methods are successfully used in practice, the size of a portfolio with thousands of assets makes optimizing them difficult, if not impossible to solve. In this article we introduce a large CVaR-based portfolio selection method by imposing weight constraints on the standard CVaR-based portfolio selection model, which effectively avoids extreme positions often emerging in traditional methods. We propose to solve the large CVaR-based portfolio model with weight constraints using penalized quantile regression techniques, which overcomes the difficulties of large scale optimization in traditional methods. We illustrate the method via empirical analysis of optimal portfolios on Shanghai and Shenzhen 300 (HS300) index and Shanghai Stock Exchange Composite (SSEC) index of China. The empirical results show that our method is efficient to solve a large portfolio selection and performs well in dispersing tail risk of a portfolio by only using a small amount of financial assets.
\end{abstract}

Keywords:

Finance, CVaR-based portfolio, risk assessment, weight constraints, quantile regression

*Corresponding author at: School of Management, Hefei University of Technology, Hefei 230009, Anhui, PR China. Tel: +86 551 62919150; fax: +86 55162905263.

Email addresses: xuqifa1975@gmail.com (Qifa Xu), ida9102@sina.com (Yingying Zhou), jiangcuixia@hfut.edu.cn (Cuixia Jiang), Keming.Yu@brunel.ac.uk (Keming $\mathrm{Yu}$ ), niu@stat.fsu.edu (Xufeng Niu) 


\section{Introduction}

Risk measure is a core research content for financial econometrics and plays an important role in the fields of portfolio investment decision, financial asset pricing and financial risk management. The classical mean-variance model proposed by Markowitz (1952) is very popular for its simplicity. In the context of the Markowitz (1952), an optimal set of weights is one in which the portfolio minimizes investment risk with an acceptable expected return. Here the variance of portfolio return is taken as a risk. To solve the Markowitz's model, it is necessary to calculate the variance-covariance matrix of all assets. The computational burden depends on the size of portfolio and a large portfolio makes optimizing them difficult, if not impossible to solve. In addition, the variance may not be an appropriate risk measure if the difference of variation of returns on upside and downside needs to be considered. Downside risk, including value at risk (VaR), conditional value at risk $(\mathrm{CVaR})$, and Expected Shortfall $(\mathrm{ES})$, is the financial risk associated with losses. Until now, VaR is a widely used risk measure and becomes a standard tool of risk management. VaR has also been successfully applied to construct mean-VaR model for portfolio selection, see Alexander and Baptista (2002), Consigli (2002), and Chuang et al. (2014) for more details. However, VaR provides non handle on the potential size of the losses beyond the VaR threshold. As an alternative risk measure, CVaR has superior properties in many respects. A big advantage of CVaR over VaR in the portfolio context is the preservation of convexity, which makes the global optimal solutions are always available, see Rockafellar and Uryasev (2000, 2002), and Quaranta and Zaffaroni (2008) for more details.

In many portfolio selection models, including mean-variance, mean-VaR and mean-CVaR, two commonly used optimization methods are quadratic programming and large-scale linear programming, and they are very complicated and time consuming, see Roman et al. (2007) and Li et al. (2012) for more details. From a decision making perspective, the optimal portfolio can be achieved by maximizing a specified expected utility defined by the familiar Lebesgue integral. Instead of Lebesgue integral, a pessimistic decision criterion in Bassett et al. (2004) can be created by using Choquet integral, permitting the probability weights associated with the least favorable outcomes to be accentuated. Then, in contrast to Markowitz's mean-variance 
portfolio allocation implemented by solving least-squares mean regression problems, this pessimistic portfolio is constructed through a standard quantile regression $(\mathrm{QR})$ technique. QR not only simplifies the numerical optimization but also provides a united framework for further promotion since it allows direct modeling of the tails of a distribution rather than the average values, please refer to $\mathrm{Xu}$ et al. (2015). Under the framework of pessimistic portfolio, the minimum CVaR-based portfolio selection model is investigated by Laurini (2007) and compared with Markowitz's mean-variance model. The empirical results show that the CVaR-based portfolios constructed using pessimistic portfolio methodology outperform both Ibovespa benchmark and optimal portfolio made using mean-variance model in terms of tail risk. As an alternative, a generalized regression method focused on superquantile is proposed by Rockafellar et al. (2014) to model CVaR directly and presents computationally tractable.

In practice, monitoring and managing a large-scale portfolio are very costing and time-consuming. It is necessary to select an appropriate size of financial assets to improve the efficiency of portfolio investment. Therefore, we aim to establish a robust portfolio with less number of financial assets to reduce trading, monitoring and research costs. Under this portfolio we intend to control the proportion of investment to avoid extreme long and short positions. To this end, it is well-known in the literature that imposing portfolio weight constraints leads to a small number of financial assets and superior performance of efficient portfolios. No-short-sale constraints are imposed by Jagannathan and Ma (2003) on the Markowitz's mean-variance model to effectively avoid the extreme and unstable asset weights. A large portfolio selection approach is proposed by Fan et al. (2012) using gross-exposure constraints and can be solved through LARS-LASSO algorithm. A penalty proportional to the sum of the absolute values of the portfolio weights is added by Brodie et al. (2009) to the objective function and leads to regularization of the optimization problem, which encourages sparse portfolio and allows accounting for transaction costs. Optimal sparse portfolios are also considered by Fastrich et al. (2012) using regularization methods. They propose a simple type of penalty that explicitly accounts financial information, which can lead to the construction of portfolios with superior out-of-sample performance and solve large portfolio selection problems. The $L_{q}$ norm constrained minimum variance portfolio is solved by the coordinate-wise algorithms proposed in Yen and Yen (2013), which has several extensions with different convex norm penalties. A unified portfolio framework incorporates Second 
order Stochastic Dominance (SSD), CVaR, and enhanced indexation, is presented by Roman et al. (2013), who find that the imposition of cardinality constraints on the SSD-based models causes to select a much lower number of stocks than the established index tracking models.

In this article, we propose a new method for large portfolio selection problems by imposing weight constraints on the standard CVaR-based portfolio selection model. As demonstrated later, our method is easy to implement via penalized quantile regression techniques. It intends to pick up a reasonable number of assets to construct a robust portfolio without too extreme positions, which has important implications for practical portfolio selection. For illustration, we apply the new method to construct optimal portfolios with assets selected from the constituent stocks in the Shanghai and Shenzhen 300 indices and Shanghai Stock Exchange Composite index of China. The empirical results show that our method outperforms some conventional methods for large portfolio selection in terms of VaR, CVaR, or Sharpe ratio.

The rest of the paper is organized as follows. In Section 2 we introduce the asset selection framework of large CVaR-based portfolio selection problem and present the regularization approaches for minimum CVaR portfolios in detail. In Section 3 we propose a procedure to solve the problem using penalized quantile regression tools. Our method is applied to real data analysis and its performance is compared with some conventional approaches in Section 4. We conclude in Section 5.

\section{Regularization approaches for minimum CVaR-based portfolio}

In this section, we introduce regularization approaches in the optimization of CVaR-based portfolio. we discuss the results and compare it with those traditional method.

\subsection{CVaR risk measure of a portfolio}

$\mathrm{CVaR}$ is a risk measure to evaluate the market risk or credit risk of a portfolio. CVaR is an extension of $\mathrm{VaR}$ and defined as the average value of a loss over a certain threshold (VaR). For a portfolio return variable $Y$ with distribution function $F$, the CVaR of $Y$ at confidence level $100(1-\tau) \%$ or quantile level $\tau \in(0,1)$ can be defined as

$$
C V a R_{\tau}=E\left[-Y \mid-Y \geq V a R_{\tau}\right]
$$


where $E\left[\cdot \mid-Y \geq V a R_{\tau}\right]$ is the conditional expectation on the condition of $\left\{-Y \geq V a R_{\tau}\right\}$. The threshold, VaR, meets

$$
\operatorname{Pr}\left[-Y \geq V a R_{\tau}\right]=\tau \text { or } \operatorname{VaR} R_{\tau}=-F^{-1}(\tau),
$$

where $\operatorname{Pr}[\cdot]$ is the probability of occurrence of an event, $F^{-1}(\cdot)$ is the inverse function of the distribution function $F$. Further, CVaR can be expressed as

$$
C V a R_{\tau}=-E\left[Y \mid Y \leq-V a R_{\tau}\right]=-\tau^{-1} \int_{0}^{\tau} F^{-1}(t) d t .
$$

From Equation (3) it can be seen that CVaR is a weighted average of a group of VaR below the threshold depending on the quantile level $\tau$.

\subsection{Standard CVaR-based portfolio selection model}

Suppose we have $p$ assets $\boldsymbol{X}=\left(X_{1}, X_{2}, \cdots, X_{p}\right)^{\prime}$ with portfolio investment scheme $\boldsymbol{\beta}=\left(\beta_{1}, \beta_{2}, \cdots, \beta_{p}\right)^{\prime}$ and get a portfolio $Y=\boldsymbol{X}^{\prime} \boldsymbol{\beta}$. An ideal portfolio investment is to get the optimized portfolio weights vector $\boldsymbol{\beta}$ through the reasonable allocation of financial assets and makes portfolio risk CVaR minimization. Here, we suppress the $\tau$ subscript in the weights vector $\boldsymbol{\beta}$ for notational convenience in spite of $\boldsymbol{\beta}$ depends on the quantile level $\tau$ for the reason that the $\mathrm{CVaR}$ is quantile dependent. The conventional CVaR-based portfolio selection model is often formulated as

$$
\left\{\begin{array}{l}
\min _{\boldsymbol{\beta}} C V a R_{\tau}(Y), \\
\text { s.t. } \mathbf{1}^{\prime} \boldsymbol{\beta}=1
\end{array}\right.
$$

where the normalization constraint on weights is written as $\mathbf{1}^{\prime} \boldsymbol{\beta}=1$, with the $p \times 1$ vector $\mathbf{1}=(1,1, \cdots, 1)^{\prime}$.

The standard CVaR-based portfolio selection model in Equation (4) is an optimization problem and can be solved by linear programming or nonsmooth programming, see Rockafellar and Uryasev (2000) for more details. In practice, the integration in Equation (3) often takes discretization and the solution depends on a scenario-based method by sampling the probability distribution of $Y$. In addition, without no-short-sell constraint the CVaRbased portfolio selection model often contains extreme positive and negative weights, for example $\beta_{i}=+800 \%, \beta_{j}=-700 \%$. These extreme weights will no doubt increase transaction costs. 


\subsection{CVaR-based portfolio selection model with weight constraints}

Inspired by Jagannathan and Ma (2003), Brodie et al. (2009), and Fan et al. (2012), we propose a robust large CVaR-based portfolio selection model by imposing weight constraints on the standard CVaR-based portfolio selection model. We consider a more general weight constraint

$$
\sum_{i=1}^{p} \operatorname{Pen}\left(\beta_{i}\right) \leq s
$$

where $s$ is the regularization parameter, $\operatorname{Pen}(\cdot)$ is a general penalty function that allow shrinking the components in $\boldsymbol{\beta}$ to exactly zero. The penalty allows simultaneous asset selection and portfolio weight estimation. We add Equation (5) to Equation (4) and get a large CVaR-based portfolio selection model with weight constraints as,

$$
\begin{cases}\min _{\boldsymbol{\beta}} & C V a R_{\tau}(Y), \\ \text { s.t. } & \mathbf{1}^{\prime} \boldsymbol{\beta}=1, \\ & \sum_{i=1}^{p} \operatorname{Pen}\left(\beta_{i}\right) \leq s .\end{cases}
$$

It is clear that the optimal portfolio result depends on the value of $s$. Generally, weaker constraint induced by the greater value of $s$, will lead to select more assets. When $s=+\infty$, the constraint $\sum_{i=1}^{p} \operatorname{Pen}\left(\beta_{i}\right) \leq+\infty$ does not work and it degenerates into the standard CVaR-based portfolio selection model. Thus, the large CVaR-based portfolio selection model with weight constraints is a general one including the standard CVaR-based portfolio selection model as a special case when $s=+\infty$.

The large CVaR-based portfolio selection model with weight constraints has an equivalent form

$$
\left\{\begin{array}{l}
\min _{\boldsymbol{\beta}} C V a R_{\tau}(Y)+\lambda \sum_{i=1}^{p} \operatorname{Pen}\left(\beta_{i}\right), \\
\text { s.t. } \mathbf{1}^{\prime} \boldsymbol{\beta}=1,
\end{array}\right.
$$

where $\lambda$ is a penalty parameter corresponding to $s$. The penalty term:

$\lambda \sum_{i=1}^{p} \operatorname{Pen}\left(\beta_{i}\right)$ mainly prevents some extreme portfolio weights, and encourages shrinkage in estimation to construct sparse portfolios. 
Many penalty forms have emerged in the literature, such as LASSO or $L_{1^{-}}$ norm penalty (Tibshirani (1996)), SCAD penalty (Softly Clipped Absolute Deviation, Fan and Li (2001)), Adaptive LASSO (Zou (2006)) and elastic net constraint (Zou and Hastie (2005)). Each penalty form renders a portfolio selection scheme. This paper focuses on SCAD penalty since it has an oracle properties. The SCAD penalty is formulated as follows,

$$
\begin{aligned}
\lambda \sum_{i=1}^{p} \operatorname{Pen}\left(\beta_{i}\right) & =\sum_{i=1}^{p}\left\{\lambda\left|\beta_{i}\right| \cdot I\left(\left|\beta_{i}\right| \leq \lambda\right)\right. \\
& \left.+\frac{-\beta_{i}^{2}+2 \alpha \lambda\left|\beta_{i}\right|-\lambda^{2}}{2(\alpha-1)} \cdot I\left(\lambda<\left|\beta_{i}\right| \leq \alpha \lambda\right)+\frac{(\alpha+1) \lambda^{2}}{2} \cdot I\left(\alpha \lambda<\left|\beta_{i}\right|\right)\right\},
\end{aligned}
$$

where $I(\cdot)$ is an indicator function that takes on the value one whenever its argument is true and zero otherwise; $\alpha(\alpha>2)$ is a tuning parameter. It is well known that SCAD is continuous and singular at the origin. SCAD penalizes large coefficients equally and has no bias. By taking advantage of the SCAD penalty, our aim is to provide an efficient method of constructing a sparse and stable portfolio from a large financial assets.

\section{Algorithm for CVaR-based portfolio selection}

In this section, we provide a algorithm for CVaR-based portfolio selection.

\subsection{Estimation of an optimal portfolio}

For a portfolio return $Y$, assume that $E Y=\mu$ exists. By deducing from the Theorem 2 in Bassett et al. (2004), we have

$$
C V a R_{\tau}=\tau^{-1} \min _{\xi} E \rho_{\tau}(Y-\xi)-\mu,
$$

where $\xi$ is the $\tau$ th quantile of $Y$, and $\rho_{\tau}(u)=u(\tau-I(u<0))$ is the check function defined in Koenker and Bassett (1978). Equation (9) bridges the gap between CVaR risk measure and objective function of quantile regression, and can be used to estimate $\mathrm{CVaR}$ and implement portfolio selection.

For a portfolio $Y=\boldsymbol{X}^{\prime} \boldsymbol{\beta}$ composed of $p$ assets $\boldsymbol{X}=\left(X_{1}, X_{2}, \cdots, X_{p}\right)^{\prime}$ with portfolio weights $\boldsymbol{\beta}=\left(\beta_{1}, \beta_{2}, \cdots, \beta_{p}\right)^{\prime}$, we have $\boldsymbol{X}^{\prime} \boldsymbol{\beta}=X_{1}-\sum_{j=2}^{p}\left(X_{1}-X_{j}\right) \beta_{j}$ under the constraint: $\mathbf{1}^{\prime} \boldsymbol{\beta}=1$. Using the derived relationship in Equation 
(9), we convert the optimizing problem of Equation (7) to a penalized quantile regression problem,

$$
\min _{\boldsymbol{\beta}, \xi} E \rho_{\tau}\left(X_{1}-\sum_{j=2}^{p} \tilde{X}_{j} \beta_{j}-\xi\right)+\lambda \sum_{i=1}^{p} \operatorname{Pen}\left(\beta_{i}\right),
$$

where $\tilde{X}_{j}=X_{1}-X_{j}(j=2,3, \cdots, p)$. This is a standard penalized linear quantile regression since $X_{1}$ denotes response, $\tilde{X}_{2}, \tilde{X}_{3}, \cdots, \tilde{X}_{p}$ are predictors, and $\xi$ denotes a constant. Note that the factor $\tau$ in Equation (9) can be absorbed into the Lagrange multiplier $\lambda$, and the term $\mu$ can be neglected since the mean is a given constant. We first solve the coefficients $\tilde{\boldsymbol{\beta}}=$ $\left(\beta_{2}, \beta_{3}, \cdots, \beta_{p}\right)^{\prime}$ by penalized quantile regression analysis, and then get the optimal weights $\boldsymbol{\beta}$ through the relationship: $\boldsymbol{\beta}=\left(1-\beta_{2}-\beta_{3}-\cdots-\beta_{p}, \tilde{\boldsymbol{\beta}}^{\prime}\right)^{\prime}=$ $\left(1-\beta_{2}-\beta_{3}-\cdots-\beta_{p}, \beta_{2}, \cdots, \beta_{p}\right)^{\prime}$. Since the penalized quantile regression approach has the ability of handling millions of variables, our portfolio method can be applied to large portfolio selection. In addition, our method is a robustness one because it is not necessary to specify a particular distribution in quantile regression analysis.

The objective function in Equation (10) is composed of the loss function:

$E \rho_{\tau}\left(X_{1}-\sum_{j=2}^{p} \tilde{X}_{j} \beta_{j}-\xi\right)$ and the penalty term: $\lambda \sum_{i=1}^{p} \operatorname{Pen}\left(\beta_{i}\right)$. After solving the optimal portfolio weights $\boldsymbol{\beta}$, we substitute $\boldsymbol{\beta}$ into the loss function and get the minimum CVaR risk of an optimal portfolio through Equation (9). Penalized quantile regression methods, such as $L_{1}$-norm quantile regression of Li and Zhu (2008), are often used to solve this problem. In this paper, we employ the SCAD penalty and the quantile regression approach of $\mathrm{Wu}$ and Liu (2009).

\subsection{Selection of tuning parameters}

To implement our method mentioned above, we need to choose twodimensional tuning parameter $\boldsymbol{\theta} \equiv(\lambda, \alpha)^{\prime}$ in the SCAD penalty. The first component $\lambda$ corresponds to the regularization parameter that drives the relevance of the SCAD penalty, which must be nonnegative. The second component $\alpha$ corresponds to quadratic spline function with knots for the $\mathrm{S}$ CAD penalty, which must be greater than two. In practice, we could search the best pair $(\lambda, \alpha)$ over the two-dimensional grids using some criteria, such as cross-validation and information criterion. To this end, we use the modified Bayesian Information Criterion (BIC) introduced by Lv et al. (2014) 
and Lee et al. (2014) as follows,

$$
\begin{aligned}
\hat{\boldsymbol{\theta}} & =\arg \min _{\boldsymbol{\theta}} B I C(\boldsymbol{\theta}) \\
& =\arg \min _{\boldsymbol{\theta}} \ln \left(\sum_{i=1}^{n} \rho_{\tau}\left(x_{i, 1}-\sum_{j=2}^{p} \tilde{x}_{i, j} \hat{\beta}_{j, \boldsymbol{\theta}}-\hat{\xi}_{\boldsymbol{\theta}}\right)\right)+d f \cdot \frac{\ln n}{2 n} \cdot \ln p
\end{aligned}
$$

where $x_{1,1}, x_{2,1}, \cdots, x_{n, 1}$ is a random sample of $X_{1}, \tilde{x}_{1, j}, \tilde{x}_{2, j}, \cdots, \tilde{x}_{n, j}$ is a random sample of $\tilde{X}_{j}$ for $j=2,3, \cdots, p$, and $d f$ is the effective dimension of fitting model. Additionally, $d f=|E|$ indicates the number of points in the set $E$ with $E=\left\{j: \hat{\beta}_{j, \boldsymbol{\theta}} \neq 0,2 \leq j \leq p\right\}$. The performance of selection criterion in Equation (11) is illustrated promising via some simulated examples and a real data analysis.

\section{Simulation studies}

In this section, we demonstrate the robustness and superiority of the proposed method through Monte Carlo simulations. We also present cases of CVaR-based portfolio that can not be efficiently solved by other method but can be tackled by the proposed one.

\subsection{Simulation data}

The classical three-factor model of Fama and French (1993) is often used to predict stock returns. Let the excessive return $X_{i}(i=1,2, \cdots, p)$ be determined by a three-factor model. We combine them and rewrite the FamaFrench three-factor model in the matrix form

$$
\underset{p \times T}{\boldsymbol{X}}=\underset{p \times 3}{\boldsymbol{B}} \underset{3 \times T}{\boldsymbol{f}}+\underset{p \times T}{\boldsymbol{\epsilon}}
$$

where $p$ is the number of assets, $T$ is the time length of observations; $\boldsymbol{B}$ is the factor loading coefficient matrix, $\boldsymbol{f}$ is the three factors matrix, $\boldsymbol{\epsilon}$ is an error matrix. Throughout this simulation, we assume that $E(\boldsymbol{\epsilon} \mid \boldsymbol{f})=\mathbf{0}$ and $\operatorname{cov}(\boldsymbol{\epsilon} \mid \boldsymbol{f})=\operatorname{diag}\left(\sigma_{1}^{2}, \sigma_{2}^{2}, \cdots, \sigma_{N}^{2}\right)$.

To generate the $T$-period returns of $p$ assets, we use the parameters, $\boldsymbol{\mu}_{\boldsymbol{B}}, \boldsymbol{\Sigma}_{\boldsymbol{B}}, \boldsymbol{\mu}_{\boldsymbol{f}}$ and $\boldsymbol{\Sigma}_{\boldsymbol{f}}$, specified in Talbe 1 of Fan et al. (2012). The whole simulation process is described as follow 4 steps: 
- Step 1: generate $\boldsymbol{B}$ and $\boldsymbol{f}$. First, the factor loadings in $\boldsymbol{B}$ are drawn from the trivariate normal distribution $N\left(\boldsymbol{\mu}_{\boldsymbol{B}}, \boldsymbol{\Sigma}_{\boldsymbol{B}}\right)$ with the parameters

$$
\boldsymbol{\mu}_{B}=\left(\begin{array}{l}
0.7828 \\
0.5180 \\
0.4100
\end{array}\right), \quad \boldsymbol{\Sigma}_{B}=\left(\begin{array}{rrr}
0.02914 & 0.02387 & 0.01018 \\
0.02387 & 0.05395 & -0.00696 \\
0.01018 & -0.00696 & 0.08685
\end{array}\right) .
$$

Second, the returns of the three factors over $T$ periods in $\boldsymbol{f}$ are randomly generated from another trivariate normal distribution $N\left(\boldsymbol{\mu}_{\boldsymbol{f}}, \boldsymbol{\Sigma}_{\boldsymbol{f}}\right)$ with the parameters

$$
\boldsymbol{\mu}_{f}=\left(\begin{array}{l}
0.02355 \\
0.01298 \\
0.02071
\end{array}\right), \quad \boldsymbol{\Sigma}_{f}=\left(\begin{array}{rrr}
1.2507 & -0.0350 & -0.2042 \\
-0.0350 & 0.3156 & -0.0023 \\
-0.2042 & -0.0023 & 0.1930
\end{array}\right) .
$$

- Step 2: generate random error. For each $i(i=1,2, \cdots, p)$, the levels of $\epsilon_{i}$ in $\boldsymbol{\epsilon}$ are generated from the gamma distribution with the shape parameter 3.3586 and the scale parameter 0.1876 .

- Step 3: generate T-period returns of $\boldsymbol{X}$ through Equation (12).

- Step 4: repeat the above three steps 999 times.

\subsection{Comparison results}

In the mean-CVaR portfolio, a commonly used method is the scenariobased one provided by Rockafellar and Uryasev (2000). They design a auxiliary function to solve the mean-CVaR portfolio and deduce that minimum of CVaR can be optimized through optimization of the auxiliary function with respect to the weights and VaR. The scenario-based method (Scen-CVaR) can be implemented via the 'fPortfolio' package in R3.2.0, and we specify the parameter by constraints='Short' to allow short sale. We compare it with our proposed CVaR-based portfolio model with SCAD penalty (SCADCVaR), and use the equal weights portfolio (EWP) model as a benchmark. All numerical calculations are carried out on an AMD FX(tm)-8350 (Eightcore $4.0 \mathrm{GMHz}$ ) processors with $32 \mathrm{~GB}$ RAM.

We consider different combination of $T$ and $p$ and report the comparison results in Table 1 and Table 2 for $T>p$ and $T<p$ respectively. Due to the limited space, the optimal values of tuning parameter $\boldsymbol{\theta}$ is not presented here, and we only report the performance of SCAD-CVaR at $\tau=0.05$ since the results are very similar at the other quantiles. 
As seen from Table 1, the computation time of Scen-CVaR method mainly depends on the time length of observations $T$ and increaments significantly with $T$ increasing. We find that the Scen-CVaR method is failed to solve the mean-CVaR portfolio due to the limited memory if $T$ is equal to or larger than 15120. While in this case, the proposed SCAD-CVaR method still works efficiently. Both of the Scen-CVaR method and the SCAD-CVaR method are superior to the EWP method in terms of the value of CVaR, and the SCAD-CVaR method has the best performance.

The main reason behind the failure of Scen-CVaR method is that we must solve a large scale optimization problem when a large amount of scenarios is necessary. Actually, the auxiliary function is a convex function of the weights, which cause the solution of the mean-CVaR portfolio be a typical convex optimization problem. However, it is difficult or impossible to determine the joint density function of $\boldsymbol{X}$ in the auxiliary function. Instead, we approximate the joint density function by using the empirical distribution function of $\boldsymbol{X}$ based on the available scenarios. Therefore, the practical solution depends on the scenario-based method by sampling the probability distribution of $\boldsymbol{X}$, which may yield a large scale optimization problem in the case of large number of observations in $\boldsymbol{X}$.

In Table 2, we find that all values of CVaR obtained through the ScenCVaR method are negative and some of them are too strange to be trusted, which implies that the Scen-CVaR method is also failed in the case of $T<p$. It seems that it is not sufficient to approximate the joint distribution of $\boldsymbol{X}$ by using a small number of observations when $p$ is large. Therefore, the ScenCVaR method will create big errors in this case. However, the SCAD-CVaR method can still work through variable selection of SCAD penalty and is able to obtain smaller value of CVaR. Its computation time mainly depends on the number of assets $p$.

To summarize, the SCAD-CVaR method takes a penalized quantile regression as core technique, which makes it a robustness method to solve the mean-CVaR portfolio. It does not only outperform the Scen-CVaR method and the EWP method in terms of value of CVaR, but also can tackle some vast portfolio selection that can not be efficiently solved by traditional method such as the Scen-CVaR method. 
Table 1

Performance of two methods for different cases $(T>p)$.

\begin{tabular}{|c|c|c|c|c|c|c|c|}
\hline \multirow{2}{*}{ Cases } & \multirow{2}{*}{ Method } & \multicolumn{2}{|c|}{ Time } & \multicolumn{2}{|c|}{$90 \%-\mathrm{CVaR}$} & \multicolumn{2}{|c|}{$99 \%-\mathrm{CVaR}$} \\
\hline & & Avg. & S.D. & Avg. & S.D. & Avg. & S.D. \\
\hline \multirow[t]{3}{*}{$T=252, p=10$} & EWP & 0.000 & 0.000 & 1.103 & 0.147 & 1.820 & 0.279 \\
\hline & Scen-CVaR & 0.038 & 0.020 & 0.348 & 0.181 & 0.355 & 0.186 \\
\hline & SCAD-CVaR & 0.121 & 0.025 & 0.310 & 0.126 & 0.344 & 0.176 \\
\hline \multirow{3}{*}{$T=252, p=50$} & EWP & 0.000 & 0.000 & 1.109 & 0.103 & 1.843 & 0.200 \\
\hline & Scen-CVaR & 0.073 & 0.035 & 0.139 & 0.050 & 0.169 & 0.126 \\
\hline & SCAD-CVaR & 0.443 & 0.056 & 0.125 & 0.052 & 0.153 & 0.106 \\
\hline \multirow[t]{3}{*}{$T=2520, p=10$} & EWP & 0.000 & 0.000 & 1.109 & 0.109 & 1.885 & 0.168 \\
\hline & Scen-CVaR & 2.935 & 0.185 & 0.488 & 0.195 & 0.530 & 0.224 \\
\hline & SCAD-CVaR & 0.295 & 0.015 & 0.431 & 0.108 & 0.481 & 0.151 \\
\hline \multirow[t]{3}{*}{$T=2520, p=50$} & EWP & 0.000 & 0.001 & 1.111 & 0.054 & 1.891 & 0.097 \\
\hline & Scen-CVaR & 3.510 & 0.104 & 0.171 & 0.119 & 0.217 & 0.136 \\
\hline & SCAD-CVaR & 1.059 & 0.059 & 0.145 & 0.054 & 0.192 & 0.090 \\
\hline \multirow[t]{3}{*}{$T=7560, p=10$} & EWP & 0.000 & 0.000 & 1.146 & 0.129 & 1.962 & 0.213 \\
\hline & Scen-CVaR & 21.032 & 0.290 & 0.662 & 0.119 & 0.683 & 0.396 \\
\hline & SCAD-CVaR & 0.718 & 0.061 & 0.488 & 0.124 & 0.526 & 0.244 \\
\hline \multirow[t]{3}{*}{$T=7560, p=50$} & EWP & 0.000 & 0.001 & 1.110 & 0.024 & 1.907 & 0.044 \\
\hline & Scen-CVaR & 27.466 & 0.375 & 0.239 & 0.136 & 0.323 & 0.126 \\
\hline & SCAD-CVaR & 2.558 & 0.115 & 0.139 & 0.016 & 0.182 & 0.037 \\
\hline \multirow[t]{3}{*}{$T=15120, p=10$} & EWP & 0.000 & 0.000 & 1.153 & 0.129 & 1.950 & 0.222 \\
\hline & Scen-CVaR & NA & NA & NA & NA & NA & NA \\
\hline & SCAD-CVaR & 1.490 & 0.277 & 0.479 & 0.125 & 0.605 & 0.218 \\
\hline \multirow[t]{3}{*}{$T=15120, p=50$} & EWP & 0.000 & 0.001 & 1.117 & 0.019 & 1.891 & 0.052 \\
\hline & Scen-CVaR & NA & NA & NA & NA & NA & NA \\
\hline & SCAD-CVaR & 5.440 & 0.377 & 0.144 & 0.017 & 0.186 & 0.022 \\
\hline
\end{tabular}

NOTE: 1) Scen-CVaR denotes the secnario-based method proposed by Rockafellar and Uryasev (2000), and SCAD-CVaR is our proposed method; 2) Time denotes time cost in seconds; 3) Avg. denotes the average value, and S.D. denotes the standard deviation; 4) NA denotes 'not available' in that the computation is out of memory. 
Table 2

Performance of two methods for different cases $(T<p)$.

\begin{tabular}{|c|c|c|c|c|c|c|}
\hline \multirow{2}{*}{ Cases } & \multirow{2}{*}{ Method } & \multicolumn{2}{|c|}{ Time } & $90 \%-C V a R$ & \multicolumn{2}{|c|}{ 99\%-CVaR } \\
\hline & & Avg. & S.D. & Avg. S.D. & Avg. & S.D. \\
\hline \multirow[t]{3}{*}{$T=126, p=500$} & EWP & 0.000 & 0.000 & 1.0820 .161 & 1.744 & 0.332 \\
\hline & Scen-CVaR & 0.337 & 0.047 & -0.3840 .081 & -22.563 & 54.903 \\
\hline & SCAD-CVaR & 32.788 & 1.031 & 0.1230 .022 & 0.154 & 0.081 \\
\hline \multirow{3}{*}{$T=126, p=1000$} & EWP & 0.001 & 0.004 & 1.0600 .134 & 1.755 & 0.347 \\
\hline & Scen-CVaR & 1.410 & 0.083 & -0.3800 .073 & -193.938 & 772.89 \\
\hline & SCAD-CVaR & 530.889 & 78 & 0.1140 & 0.133 & 0.099 \\
\hline \multirow[t]{3}{*}{$T=126, p=1500$} & EWP & 0.000 & 0.000 & 1.5990 .082 & 2.533 & 0.316 \\
\hline & Scen-CVaR & 2.140 & 0.170 & -0.4190 .087 & -10.571 & 13.170 \\
\hline & SCAD-C & 1329.648 & 84.611 & 0.1130 .016 & 0.151 & 0.084 \\
\hline \multirow[t]{3}{*}{$T=252, p=500$} & EWP & 0.000 & 0.000 & 1.1230 .127 & 1.960 & 0.296 \\
\hline & Scen-C & 1.142 & 0.192 & -0.3960 .048 & -17.381 & 21.991 \\
\hline & SCAD- & 40.772 & 0.792 & 0.1280 .010 & 0.146 & 0.040 \\
\hline \multirow[t]{3}{*}{$T=252, p=1000$} & EWP & 0.000 & 0.000 & 1.0790 .143 & 1.931 & 0.266 \\
\hline & Scen-CV & 2.852 & 0.089 & -0.4170 .083 & -11.249 & 10.845 \\
\hline & $\mathrm{SCA}$ & 566.234 & 27.877 & 0.1340 .025 & 0.149 & 0.036 \\
\hline \multirow[t]{3}{*}{$T=252, p=1500$} & EWP & 0.000 & 0.000 & 1.5250 .153 & 2.761 & 0.321 \\
\hline & & 5.612 & 0.064 & -0.4630 .057 & -160.194 & 273.501 \\
\hline & SCAD-CVaR & 865.376 & 1.054 & 0.1160 .016 & 0.188 & 0.053 \\
\hline
\end{tabular}

NOTE: The same as in Table 1. 


\section{Empirical applications}

In this section, we use two practical portfolio selections to illustrate the efficacy of our proposed method.

\subsection{Real examples}

We consider the construction of optimal sparse and stable portfolios with assets selected from the constituent stocks in two stock indices of China: the Shanghai and Shenzhen 300 (HS300) index and the Shanghai Stock Exchange Composite (SSEC) index.

The data comes from GTA data service center, CITIC Index Co., Ltd. website and Finance Google website. We use daily observations of the constituent stocks and calculate their returns using difference of logarithms, i.e., $r_{t}=100 \times\left(\ln\right.$ price $_{t}-\ln$ price $\left._{t-1}\right)$, where price $_{t}$ is the closing stock price. The sample period spans from Aug 14, 2012 to Dec 18, 2015 for HS300 and from Jan 6, 2009 to May 5, 2016 for SSEC. Note that the 300 constituent stocks in HS300 are always changing in the sample period since the sample is adjusted every half year but the number of adjusted sample stocks is uncertain. We intersect those stocks and ultimately keep $p=227$ constituent stocks for HS300. As the SSEC situation is similar to the case of HS300, we perform the same procedure to get $p=834$ constituent stocks for SSEC.

To show the performance of our method, we consider two cases: $n>p$ and $n<p$. In the case of HS300, one sample runs from Aug 14, 2012 to Dec 18, 2015 with the total sample size 835 for $n>p$, while another sample runs from Mar 24, 2015 to Dec 18, 2015 with the total sample size 185 for $n<p$. In the case of SSEC, we take the sample period from Jan 6, 2009 to May 5, 2016 with sample size 1812 for $n>p$ and from Nov 4, 2013 to May 5, 2016 with sample size 612 for $n<p$.

\subsection{Portfolio analysis}

\subsection{1. $n>p$ case}

We implement the CVaR-based portfolio selection model with weight constraints using SCAD penalized quantile regression at three quantiles $\tau=$ $0.01,0.05,0.10$. The optimal values of $(\lambda, \alpha)$ and its corresponding BICs are reported in Table 3. It is clear that the values of $\alpha$ at different quantile are very close to 3.7, which is recommended by Fan and Li (2001). The solution paths and the optimal weights are shown from Fig. 1 to Fig. 3 for HS300 and Fig. 4 to Fig. 6 for SSEC. Take Fig. 1 as an example, it shows the solution 
path of the portfolio weights and the value of penalty parameter determined by the modified BIC criterion. As seen from panel (a) of Fig. 1, the number of selected financial assets is gradually increasing with $\lambda$ decreasing. When $\lambda$ is 68.1 corresponding to the smallest BIC value, the optimal portfolio are constructed with 18 assets selected, and the weights are presented in panel (b) of Fig. 1. From the barplot in panel (b), there is no extreme weights, and the maximum weights of the portfolio is 0.3697 while the minimum is 0.0005. The other two figures show the similar results at quantiles 0.05 and 0.1 to the result at quantile 0.01 in Fig. 1. These results imply that our method tends to select less assets for the optimal portfolio and is able to avoid generating extreme weights in practice. In the next step, we will show the effects of risk allocation using our method.

\section{Table 3}

The optimal results of parameter selection $(n>p)$

\begin{tabular}{lccccccc}
\hline \multirow{2}{*}{$\tau$} & \multicolumn{3}{c}{ HS300 } & & \multicolumn{3}{c}{ SSEC } \\
\cline { 2 - 4 } \cline { 6 - 7 } & $\lambda$ & $\alpha$ & BIC & & $\lambda$ & $\alpha$ & BIC \\
\hline 0.01 & 0.09 & 3.72 & 6.1661 & & 0.11 & 3.73 & 7.7280 \\
0.05 & 0.08 & 3.74 & 6.3098 & & 0.14 & 3.69 & 7.1589 \\
0.10 & 0.10 & 3.69 & 5.9506 & & 0.13 & 3.71 & 7.3755 \\
\hline
\end{tabular}

For comparison, we also consider mean-variance (mean-var) model of Markowitz (1952), minimum variance (min-var) model, $L_{1}$-norm constraint on the variance-based portfolio selection method ( $L_{1}$-var) of Fan et al. (2012), and still use the equal weights portfolio (EWP) model as a benchmark. We compare the performance of the optimal portfolios constructed by our CVaRbased portfolio model with SCAD penalty (SCAD-CVaR) at three quantiles $\tau=0.01,0.05,0.10$ with those four methods.

Table 4 reports the description of weights and the change of positions for the optimal portfolio. We find that the traditional portfolio procedure tends to select more stocks to construct a portfolio, while the new portfolio method using shrinkage techniques tends to use a small amount of stocks, which is very useful to reduce the cost of supervision and transaction. These findings are very similar to those of Fan et al. (2012). In addition, our sparse portfolio is stable without extreme positive and negative weights compared with those conventional portfolio methods like mean-variance model, minimum variance model, and etc. As expected, all of the methods will spend more time to 
optimize a large portfolio selection like SSEC. In terms of time cost, our method spends a lot of time since we tune parameters: $\lambda$ and $\alpha$ by using grids search approach.

The means, standard deviations, and other characteristics of these portfolios are recorded and presented in Table 5 where VaR and CVaR are calculated at $90 \%$ and $99 \%$ confidence level. As far as standard deviation is concerned, the optimal value determined by SCAD-CVaR method is relatively large, and relative small determined by the methods of mean-var, the min-var, and the $L_{1}$-var due to variance-based optimization objectives. However, the Sharpe ratios of the SCAD-CVaR method are larger than those from conventional methods. When it comes to the VaR and CVaR risk, the SCAD-CVaR is also superior to the other methods. The empirical results indicate that both the HS300 portfolio and the SSEC portfolio can be effectively tracked through less stocks selected by our proposed portfolio method. In fact, the core technique in our method is a shrinkage and selection approach in statistics, which can select some relevant variables to enhance the prediction accuracy and interpretability of the statistical model.

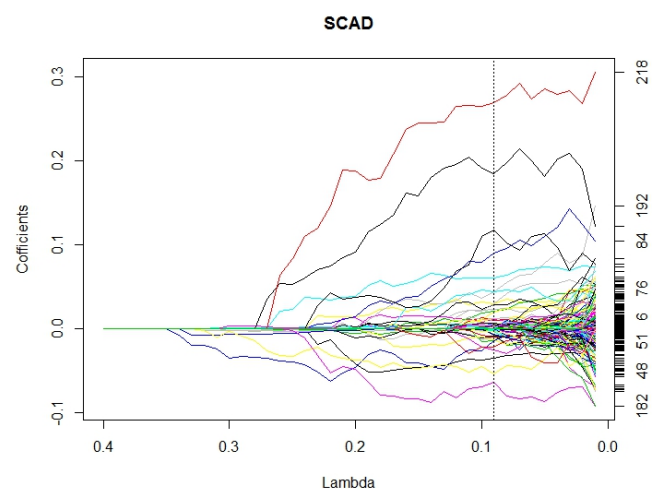

(a) Portfolio weights solution paths and BIC-based assets selection.

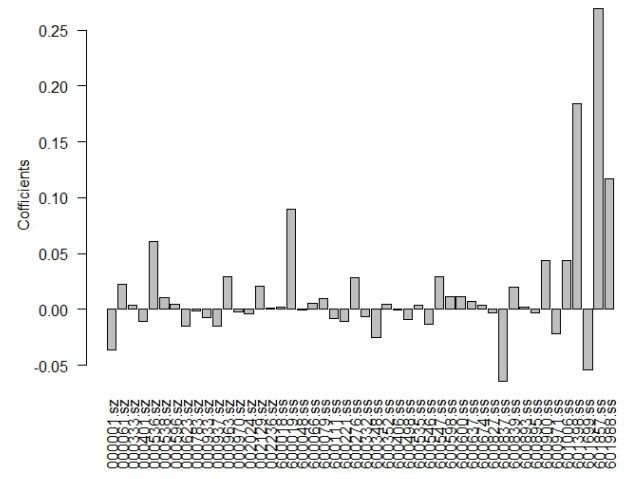

(b) Optimal portfolio weights.

Fig. 1 Portfolio weights solution paths and the optimal portfolio weights for HS300 at $\tau=0.01$ in $n>p$ case. 


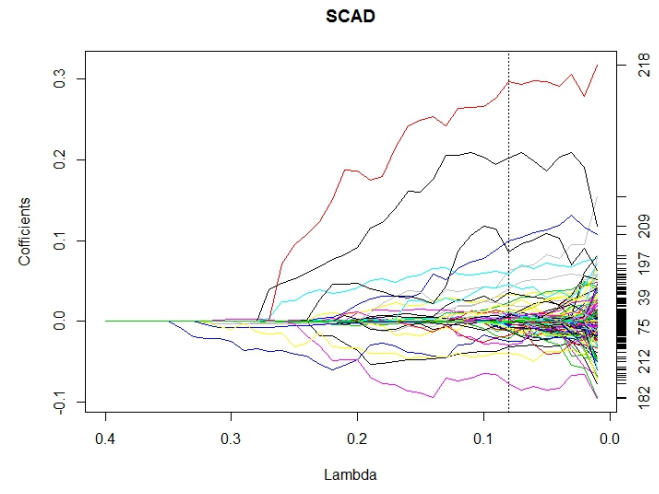

(a) Portfolio weights solution paths and BIC-based assets selection.

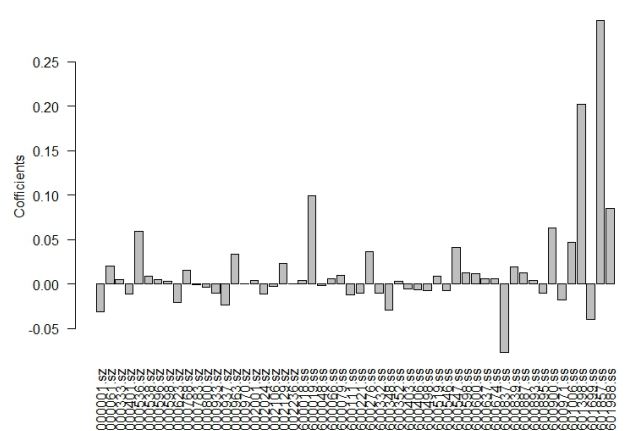

(b) Optimal portfolio weights.

Fig. 2 Portfolio weights solution paths and the optimal portfolio weights for HS300 at $\tau=0.05$ in $n>p$ case.

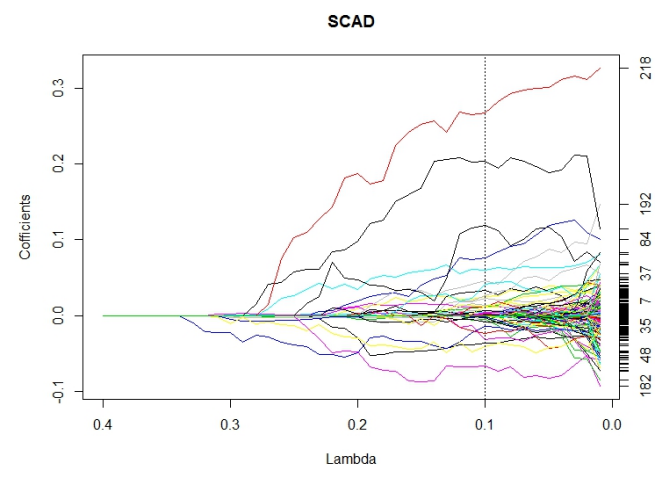

(a) Portfolio weights solution paths and BIC-based assets selection.

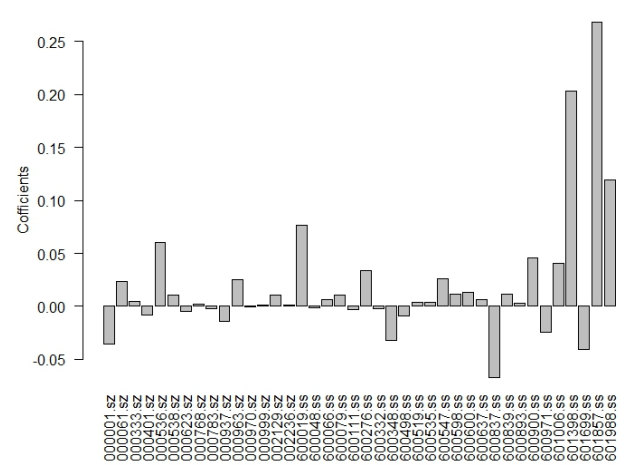

(b) Optimal portfolio weights.

Fig. 3 Portfolio weights solution paths and the optimal portfolio weights for HS300 at $\tau=0.10$ in $n>p$ case. 


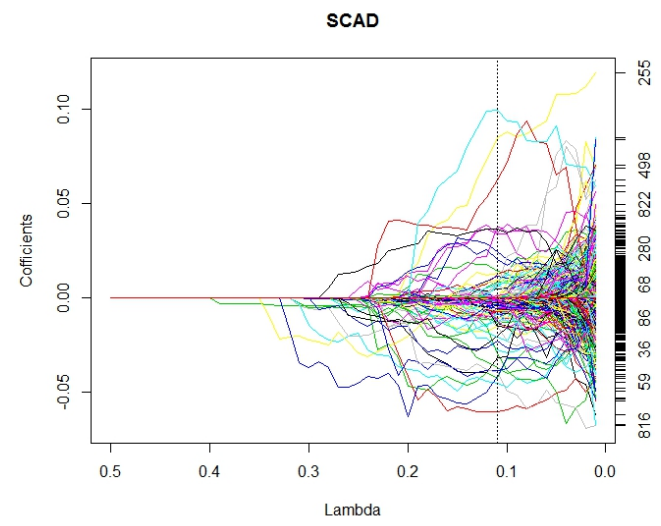

(a) Portfolio weights solution paths and BIC-based assets selection.

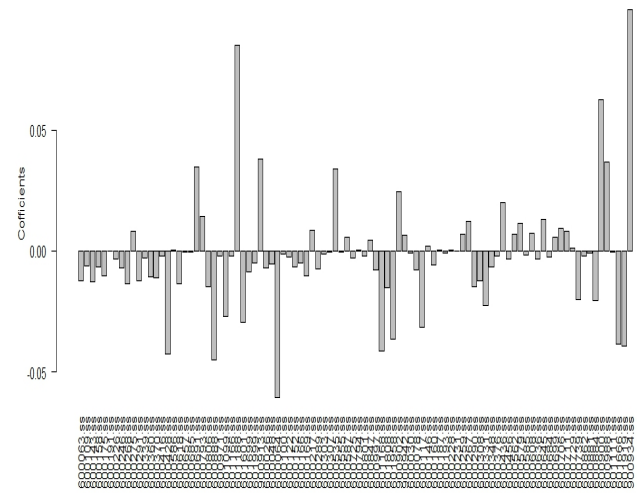

(b) Optimal portfolio weights.

Fig. 4 Portfolio weights solution paths and the optimal portfolio weights for SSEC at $\tau=0.01$ in $n>p$ case.

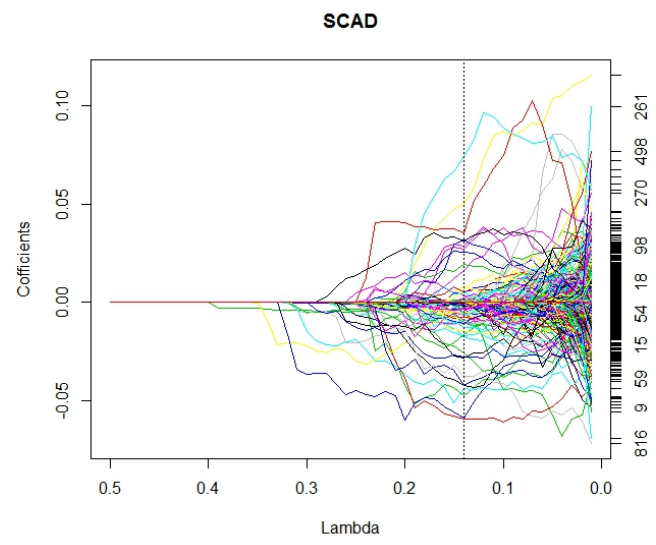

(a) Portfolio weights solution paths and BIC-based assets selection.

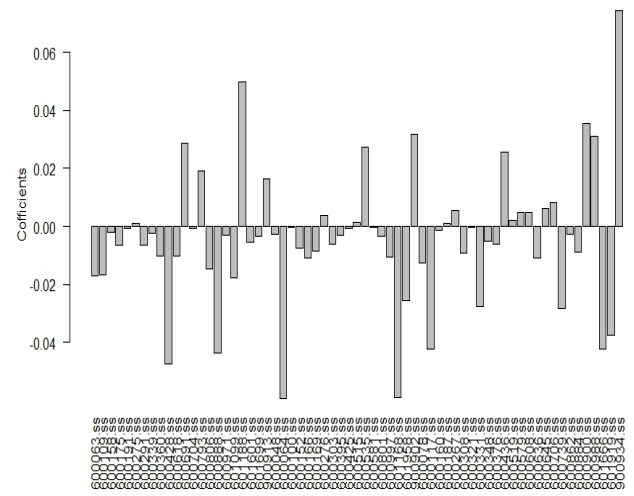

(b) Optimal portfolio weights.

Fig. 5 Portfolio weights solution paths and the optimal portfolio weights for SSEC at $\tau=0.05$ in $n>p$ case. 


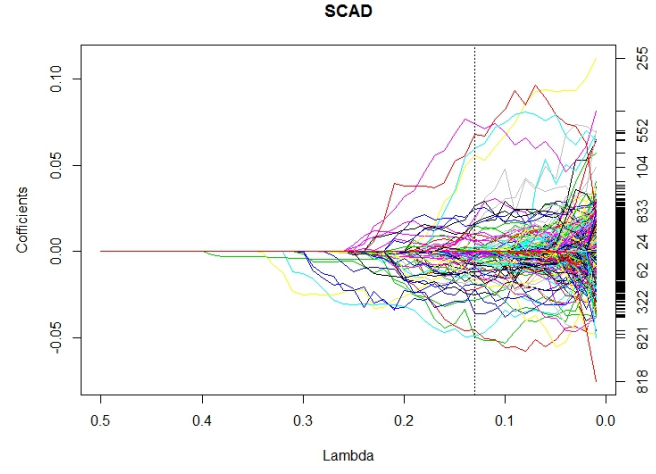

(a) Portfolio weights solution paths and BIC-based assets selection.

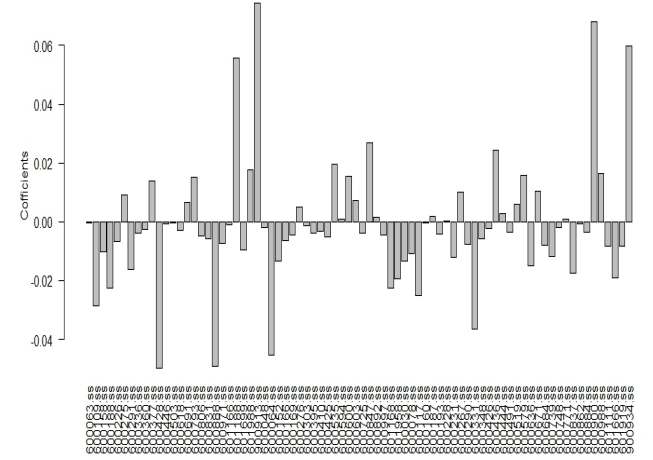

(b) Optimal portfolio weights.

Fig. 6 Portfolio weights solution paths and the optimal portfolio weights for SSEC at $\tau=0.10$ in $n>p$ case.

Table 4

Descriptions of optimal portfolios $(n>p)$

\begin{tabular}{lllrrrrrr}
\hline Indices & Method & & MaxW & MinW & \#Long & \#Short & \#Out & Time \\
\hline HS300 & EWP & - & 0.004 & 0.004 & 227 & 0 & 0 & 0.00 \\
& mean-var & - & 0.331 & 0 & 206 & 0 & 21 & 0.25 \\
& min-var & - & 0.297 & 0 & 206 & 0 & 21 & 0.20 \\
& $L_{1}$-var & - & 0.318 & -0.076 & 13 & 9 & 205 & 1.25 \\
& SCAD-CVaR & 0.01 & 0.269 & -0.064 & 27 & 21 & 179 & 7.04 \\
& & 0.05 & 0.296 & -0.077 & 31 & 23 & 173 & 7.58 \\
& & 0.10 & 0.268 & -0.067 & 26 & 14 & 187 & 7.97 \\
SSEC & EWP & - & 0.001 & 0.001 & 834 & 0 & 0 & 0.00 \\
& mean-var & - & 0.157 & 0 & 808 & 0 & 26 & 9.17 \\
& min-var & - & 0.153 & 0 & 808 & 0 & 26 & 9.11 \\
& $L_{1}$-var & - & 0.226 & -0.052 & 23 & 15 & 796 & 26.41 \\
& SCAD-CVaR & 0.01 & 0.100 & -0.060 & 29 & 67 & 738 & 1526.30 \\
& & 0.05 & 0.074 & -0.059 & 20 & 45 & 769 & 1459.76 \\
& & 0.10 & 0.074 & -0.050 & 26 & 52 & 756 & 1482.04 \\
\hline
\end{tabular}

NOTE: 1) MaxW=Max. weight, MinW=Min. weight; 2) \#Long=No. of long positions, \#Short=No. of short positions, \#Out=No. of assets outside of the optimal portfolio; 3) Time denotes time cost in seconds. 
Table 5

Returns and risks of optimal portfolios $(n>p)$

\begin{tabular}{|c|c|c|c|c|c|c|c|c|c|}
\hline \multirow{2}{*}{\multicolumn{2}{|c|}{ Indices Method }} & \multirow{2}{*}{$\tau$} & \multirow{2}{*}{ Mean } & \multirow{2}{*}{ SD } & \multicolumn{2}{|c|}{$90 \%$} & \multicolumn{2}{|c|}{$99 \%$} & \multirow{2}{*}{ Sharpe ratio } \\
\hline & & & & & VaR & CVaR & VaR & CVaR & \\
\hline \multirow[t]{7}{*}{$\overline{\mathrm{HS} 300}$} & EWP & & 0.017 & 1.149 & 2.090 & 2.855 & 2.186 & 2.907 & 0.015 \\
\hline & mean-var & & 0.017 & 0.733 & 1.610 & 2.096 & 1.702 & 2.150 & 0.023 \\
\hline & min-var & & 0.019 & 0.742 & 1.431 & 1.786 & 1.434 & 1.866 & 0.026 \\
\hline & $L_{1}$-var & & 0.019 & 0.958 & 0.898 & 1.572 & 0.988 & 1.617 & 0.019 \\
\hline & SCAD-CVaR & 0.01 & 0.267 & 2.561 & 0.514 & 1.034 & 0.575 & 1.107 & 0.104 \\
\hline & & 0.05 & 0.239 & 2.167 & 0.592 & 1.090 & 0.647 & 1.130 & 0.110 \\
\hline & & 0.10 & 0.185 & 1.711 & 0.631 & 1.085 & 0.662 & 1.117 & 0.108 \\
\hline \multirow[t]{7}{*}{ SSEC } & EWP & & 0.024 & 1.644 & 3.942 & 4.168 & 4.077 & 4.278 & 0.015 \\
\hline & mean-var & & 0.024 & 0.916 & 3.469 & 3.623 & 3.558 & 3.714 & 0.026 \\
\hline & min-var & & 0.009 & 0.910 & 2.458 & 2.935 & 2.501 & 2.992 & 0.009 \\
\hline & $L_{1}$-var & 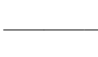 & 0.015 & 0.738 & 2.005 & 2.522 & 2.128 & 2.567 & 0.020 \\
\hline & SCAD-CVaR & 0.01 & 0.067 & 0.791 & 1.410 & 2.027 & 1.508 & 2.130 & 0.085 \\
\hline & & 0.05 & 0.065 & 0.880 & 1.256 & 1.909 & 1.306 & 1.920 & 0.074 \\
\hline & & 0.10 & 0.056 & 0.851 & 1.543 & 1.949 & 1.603 & 2.061 & 0.066 \\
\hline
\end{tabular}




\subsection{2. $n<p$ case}

In this case, we also use the SCAD-CVaR method to construct sparse and stable portfolios at three quantiles: $0.01,0.05$, and 0.10 . The optimal results of parameter selection are reported in Table $\mathbf{6}$, while the solution paths and the optimal weights are depicted in three figures (from Fig. 7 to Fig. 9 for HS300, from Fig. 10 to Fig. 12 for SSEC). The results are similar to those in the case of $n>p$, we only select 19 assets without extreme weights for the optimal portfolio at 0.01 quantile. We leave out similar conclusions from other quantile levels with aiming to save space.

\section{Table 6}

The optimal results of parameter selection $(n<p)$

\begin{tabular}{cccccccc}
\hline \multirow{2}{*}{$\tau$} & \multicolumn{3}{c}{ HS300 } & & \multicolumn{3}{c}{ SSEC } \\
\cline { 2 - 4 } \cline { 6 - 8 } & $\lambda$ & $\alpha$ & BIC & & $\lambda$ & $\alpha$ & BIC \\
\hline 0.01 & 0.11 & 3.65 & 6.5886 & & 0.18 & 3.70 & 7.5000 \\
0.05 & 0.11 & 3.68 & 6.5776 & & 0.19 & 3.76 & 7.5350 \\
0.10 & 0.12 & 3.76 & 6.4847 & & 0.19 & 3.73 & 7.6658 \\
\hline
\end{tabular}

Here, we only compare the SCAD-CVaR method with the EWP method and the $L_{1}$-var method since the mean-var and the min-var methods are invalid for the case of $n<p$. Table 7 reports the description of weights and the change of positions for the optimal portfolio for $n<p$ case. The results indicate that both $L_{1}$-var method and SCAD-CVaR method are valid to select small amount of stocks for an optimal portfolio and have a similar distribution of weights. In fact, the $L_{1}$-var method can provide sparse portfolio through LASSO mean regression of Tibshirani (1996). It is able to effectively disperse variance risk. Different from the $L_{1}$-var method, our SCAD-CVaR method brings sparsity through penalized quantile regression, which mainly focuses on tail risk. The SCAD-CVaR method can implement risk diversification by only using a small amount of stocks. As far as computation time be concerned, the $n<p$ case will cost more time than the $n>p$ case when we compare Table 7 with Table 4 .

The compared performances are reported in Table 8 and are similar to those in Table 5. Although the SCAD-CVaR method is not the best one in terms of standard deviation, it outperforms the other two methods in terms of VaR, CVaR, or Sharpe ratio. 


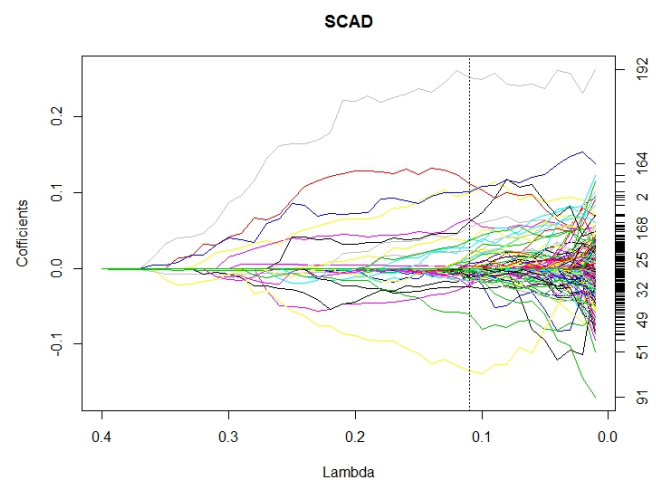

(a) Portfolio weights solution paths and BIC-based assets selection.

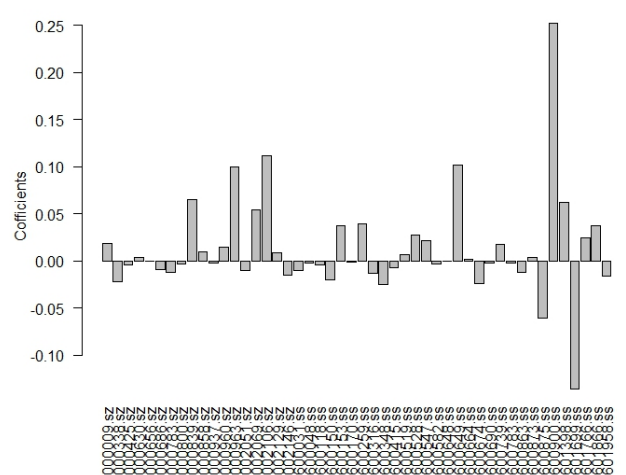

(b) Optimal portfolio weights.

Fig. 7 Portfolio weights solution paths and the optimal portfolio weights for HS300 at $\tau=0.01$ in $n<p$ case.

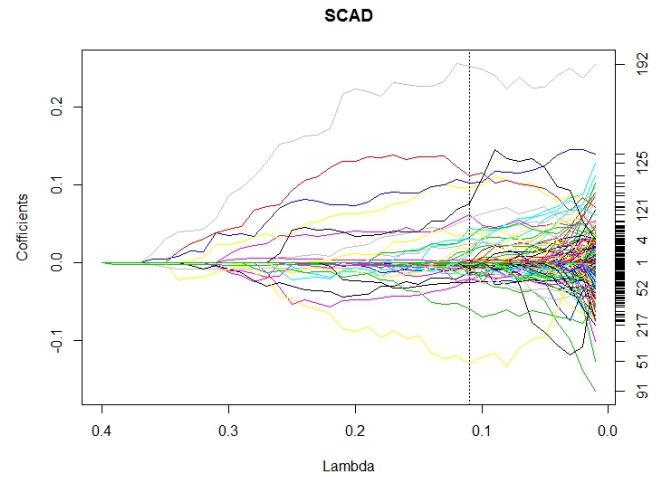

(a) Portfolio weights solution paths and BIC-based assets selection.

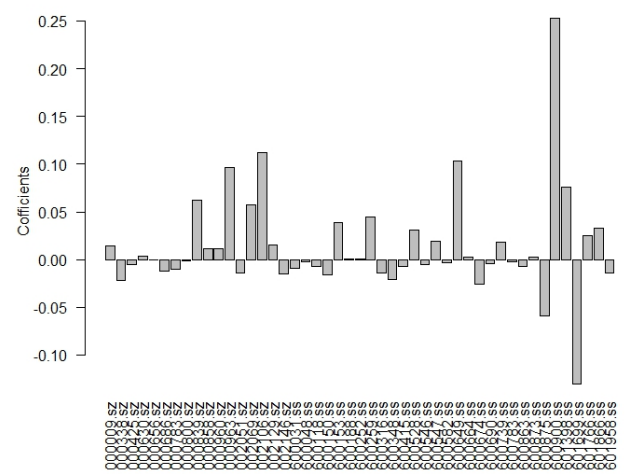

(b) Optimal portfolio weights.

Fig. 8 Portfolio weights solution paths and the optimal portfolio weights for HS300 at $\tau=0.05$ in $n<p$ case. 


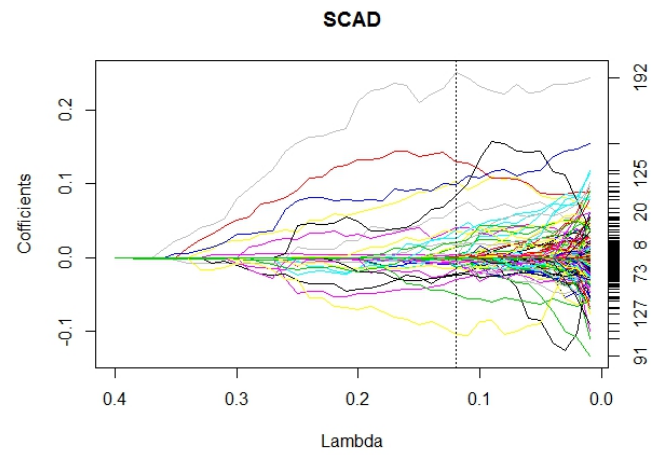

(a) Portfolio weights solution paths and BIC-based assets selection.

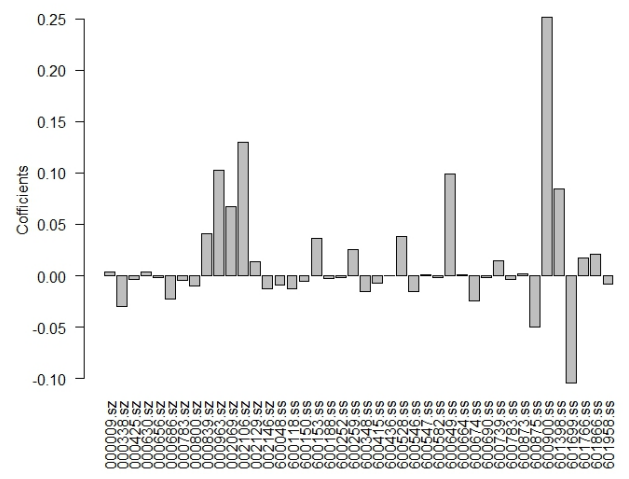

(b) Optimal portfolio weights.

Fig. 9 Portfolio weights solution paths and the optimal portfolio weights for HS300 at $\tau=0.10$ in $n<p$ case.

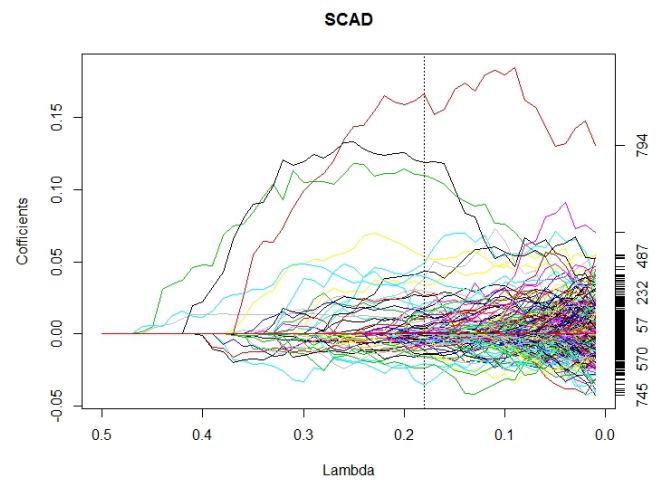

(a) Portfolio weights solution paths and BIC-based assets selection.

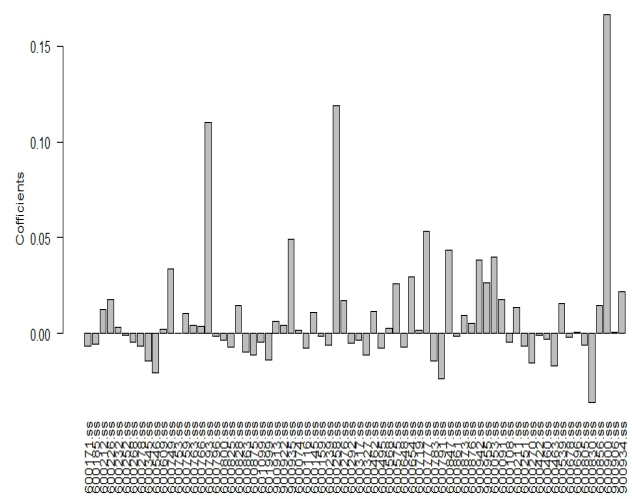

(b) Optimal portfolio weights.

Fig. 10 Portfolio weights solution paths and the optimal portfolio weights for SSEC at $\tau=0.01$ in $n<p$ case. 


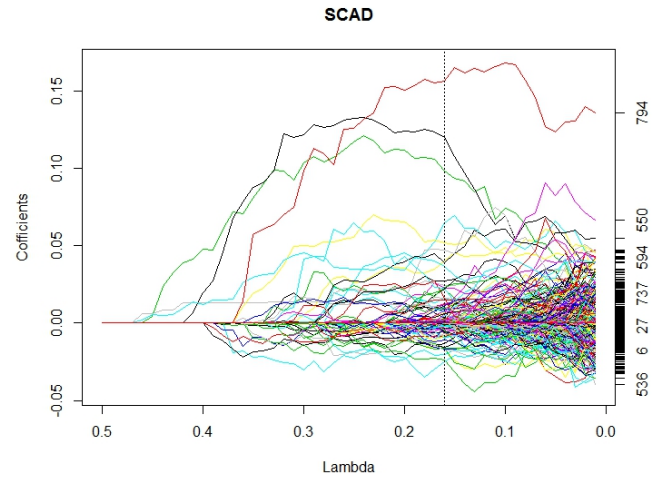

(a) Portfolio weights solution paths and BIC-based assets selection.

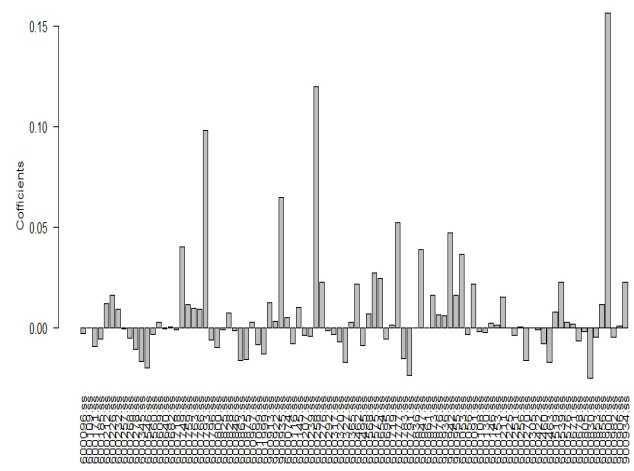

(b) Optimal portfolio weights.

Fig. 11 Portfolio weights solution paths and the optimal portfolio weights for SSEC at $\tau=0.05$ in $n<p$ case.

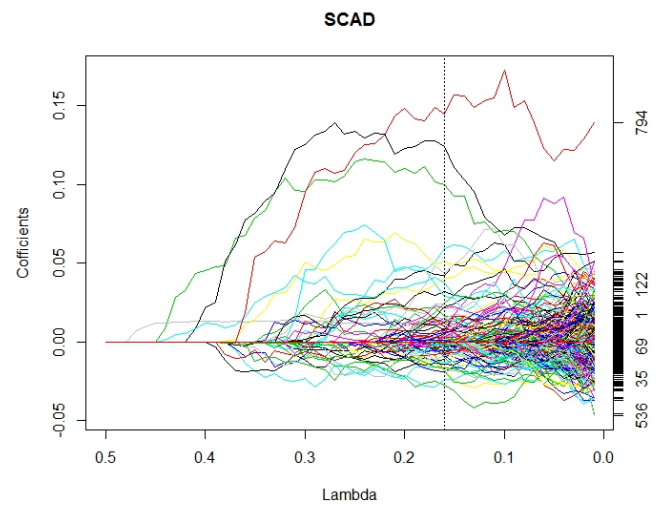

(a) Portfolio weights solution paths and BIC-based assets selection.

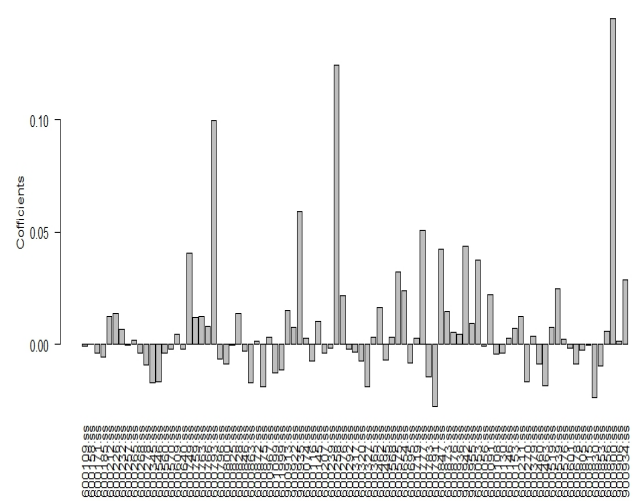

(b) Optimal portfolio weights.

Fig. 12 Portfolio weights solution paths and the optimal portfolio weights for SSEC at $\tau=0.10$ in $n<p$ case. 


\section{Table 7}

Descriptions of optimal portfolios $(n<p)$

\begin{tabular}{llrrrrrrr}
\hline Indices & Method & & \multicolumn{1}{c}{ MaxW } & MinW & \#Long & \#Short & \#Out & Time \\
\hline HS300 & EWP & - & 0.004 & 0.004 & 227 & 0 & 0 & 0.00 \\
& $L_{1}$-var & & 0.230 & -0.064 & 24 & 18 & 185 & 9.08 \\
& SCAD-CVaR & 0.01 & 0.252 & -0.136 & 22 & 26 & 179 & 24.80 \\
& & 0.05 & 0.253 & -0.13 & 21 & 26 & 180 & 24.64 \\
& & 0.10 & 0.251 & -0.104 & 20 & 22 & 185 & 22.48 \\
SSEC & EWP & & 0.001 & 0.001 & 834 & 0 & 0 & 0.00 \\
& $L_{1}$-var & & 0.188 & -0.018 & 32 & 16 & 786 & 83.18 \\
& SCAD-CVaR & 0.01 & 0.166 & -0.036 & 38 & 34 & 762 & 4285.77 \\
& & 0.05 & 0.156 & -0.025 & 45 & 49 & 740 & 4141.57 \\
& & 0.10 & 0.145 & -0.027 & 46 & 43 & 745 & 4020.99 \\
\hline
\end{tabular}

NOTE: 1) MaxW=Max. weight, MinW=Min. weight; 2) \#Long=No. of long positions, \#Short=No. of short positions, \#Out=No. of assets outside of the optimal portfolio; 3) Time denotes time cost in seconds.

\section{Table 8}

Returns and risks of optimal portfolios $(n<p)$

\begin{tabular}{|c|c|c|c|c|c|c|c|c|c|}
\hline \multirow{2}{*}{ Indices } & \multirow{2}{*}{ Method } & \multirow{2}{*}{$\tau$} & \multirow{2}{*}{ Mean } & \multirow{2}{*}{$\mathrm{SD}$} & \multicolumn{4}{|c|}{$99 \%$} & \multirow{2}{*}{-Sharpe ratio } \\
\hline & & & & & VaR & CVaR & VaR & CVaR & \\
\hline \multirow[t]{5}{*}{ HS300 } & EWP & & 0.061 & 1.293 & 2.572 & 2.863 & 2.677 & 2.915 & 0.047 \\
\hline & $L_{1}$-var & & 0.050 & 0.979 & 1.605 & 1.711 & 1.613 & 1.736 & 0.051 \\
\hline & SCAD-CVaR & 0.01 & 0.219 & 1.520 & 0.888 & 1.203 & 0.932 & 1.230 & 0.144 \\
\hline & & 0.05 & 0.184 & 1.279 & 0.654 & 1.223 & 0.717 & 1.280 & 0.144 \\
\hline & & 0.10 & 0.177 & 0.981 & 0.608 & 1.153 & 0.642 & 1.269 & 0.180 \\
\hline \multirow[t]{5}{*}{ SSEC } & EWP & 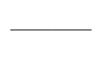 & 0.031 & 2.284 & 4.267 & 6.755 & 4.389 & 6.853 & 0.013 \\
\hline & $L_{1}$-var & - & 0.080 & 0.824 & 1.665 & 2.495 & 1.736 & 2.596 & 0.097 \\
\hline & SCAD-CVaR & 0.01 & 0.133 & 0.879 & 1.459 & 1.933 & 1.578 & 2.062 & 0.152 \\
\hline & & 0.05 & 0.131 & 0.831 & 1.237 & 1.876 & 1.334 & 1.952 & 0.157 \\
\hline & & 0.10 & 0.125 & 0.823 & 1.361 & 1.901 & 1.430 & 1.930 & 0.151 \\
\hline
\end{tabular}




\section{Conclusion}

In this paper, we propose the large CVaR-based portfolio selection model with weight constraints by imposing the weight constraints on the standard CVaR-based portfolio selection model. This new model is a general one that includes the standard CVaR-based portfolio selection model as a special case. We implement the method using penalized quantile regression tools. Our method can be used to construct sparse and stable optimal portfolios selected from large financial assets.

To illustrate the efficacy of our method, we carry out empirical analysis of HS300 and SSEC indices respectively, and then compare the performance of our method for optimal portfolios construction on the constituent stocks to some conventional methods. At least three conclusions can be drawn from the empirical results. First, our large CVaR-based portfolio selection model with weight constraints can always pick out a small amount of financial assets to form a portfolio no matter in the case of $n>p$ or $n<p$. Second, the final sparse portfolio is stable without extreme positive and negative weights. Third, our method is superior to those conventional methods, including EWP, mean-var, min-var, and $L_{1}$-var mthods, in terms of VaR, CVaR, or Sharpe ratio.

\section{Acknowledgements}

The authors would like to thank the Editor-in-Chief and two anonymous reviewers for their helpful recommendation and comments.

This work was supported by the National Social Science Foundation of PR China (15BJY008), the Humanity and Social Science Foundation of Ministry of Education of PR China ( 14YJA790015), and the Science and Technology Development Plan Project of Shandong Province (2014GGX101055).

\section{References}

Alexander, G.J., Baptista, A.M., 2002. Economic implications of using a mean-var model for portfolio selection: A comparison with mean-variance analysis. Journal of Economic Dynamics and Control 26, 1159-1193.

Bassett, G., Koenker, R., Kordas, G., 2004. Pessimistic portfolio allocation and choquet expected utility. Journal of Financial Econometrics 2, 477492 . 
Brodie, J., Daubechies, I., De Mol, C., Giannone, D., Loris, I., 2009. Sparse and stable markowitz portfolios. Proceedings of the National Academy of Sciences 106, 12267-12272.

Chuang, C.C., Wang, Y.H., Yeh, T.J., Chuang, S.L., 2014. Backtesting var in consideration of the higher moments of the distribution for minimumvariance hedging portfolios. Economic Modelling 42, 15-19.

Consigli, G., 2002. Tail estimation and meancvar portfolio selection in markets subject to financial instability. Journal of Banking \& Finance 26, $1355-1382$.

Fama, E.F., French, K.R., 1993. Common risk factors in the returns on stocks and bonds. Journal of Financial economics 33, 3-56.

Fan, J., Li, R., 2001. Variable selection via nonconcave penalized likelihood and its oracle properties. Journal of the American Statistical Association 96, 1348-1360.

Fan, J., Zhang, J., Yu, K., 2012. Vast portfolio selection with gross-exposure constraints. Journal of the American Statistical Association 107, 592-606.

Fastrich, B., Paterlini, S., Winker, P., 2012. Constructing optimal sparse portfolios using regularization methods. Computational Management Science $12,417-434$.

Jagannathan, R., Ma, T., 2003. Risk reduction in large portfolios: Why imposing the wrong constraints helps. The Journal of Finance 58, 16511684.

Koenker, R., Bassett, G.W., 1978. Regression quantiles. Econometrica 46, 33-50.

Laurini, M.P., 2007. Pessimistic preferences and portfolio allocationempirical analysis and risk management applications. Revista de Economia e Administracao 6, 347-371.

Lee, E.R., Noh, H., Park, B.U., 2014. Model selection via bayesian information criterion for quantile regression models. Journal of the American Statistical Association 109, 216-229. 
Li, J., Huang, H., Xiao, X., 2012. The sovereign property of foreign reserve investment in china: A cvar approach. Economic Modelling 29, 1524-1536.

Li, Y., Zhu, J., 2008. L1-norm quantile regression. Journal of Computational and Graphical Statistics 17, 163-185.

Lv, Y., Zhang, R., Zhao, W., Liu, J., 2014. Quantile regression and variable selection for the single-index model. Journal of Applied Statistics 41, 15651577 .

Markowitz, H., 1952. Portfolio selection. Journal of Finance 7, 77-91.

Quaranta, A.G., Zaffaroni, A., 2008. Robust optimization of conditional value at risk and portfolio selection. Journal of Banking \& Finance 32, 2046-2056.

Rockafellar, R.T., Royset, J.O., Miranda, S.I., 2014. Superquantile regression with applications to buffered reliability, uncertainty quantification, and conditional value-at-risk. European Journal of Operational Research 234, $140-154$.

Rockafellar, R.T., Uryasev, S., 2000. Optimization of conditional value-atrisk. Journal of Risk 2, 21-42.

Rockafellar, R.T., Uryasev, S., 2002. Conditional value-at-risk for general loss distributions. Journal of Banking \& Finance 26, 1443-1471.

Roman, D., Darby-Dowman, K., Mitra, G., 2007. Mean-risk models using two risk measures: a multi-objective approach. Quantitative Finance volume 7, 443-458.

Roman, D., Mitra, G., Zverovich, V., 2013. Enhanced indexation based on second-order stochastic dominance. European Journal of Operational Research 228, 273-281.

Tibshirani, R., 1996. Regression shrinkage and selection via the lasso. Journal of the Royal Statistical Society: Series B 58, 267-288.

Wu, Y., Liu, Y., 2009. Variable selection in quantile regression. Statistica Sinica 19, 801-817. 
Xu, Q., Niu, X., Jiang, C., Huang, X., 2015. The phillips curve in the us: A nonlinear quantile regression approach. Economic Modelling 49, 186-197.

Yen, Y.M., Yen, T.J., 2013. Solving norm constrained portfolio optimization via coordinate-wise descent algorithms. Computational Statistics \& Data Analysis 76, 737-759.

Zou, H., 2006. The adaptive lasso and its oracle properties. Journal of the American Statistical Association 101, 1418-1429.

Zou, H., Hastie, T., 2005. Regularization and variable selection via the elastic net. Journal of the Royal Statistical Society: Series B 67, 301-320. 